\title{
An interconnected duplicated femoral vein and its clinical significance
}

\author{
A.A. Khan, A. Hassan, M.A. Asari, N. Aiman \\ Department of Anatomy, School of Medical Sciences, Universiti Sains Malaysia, Kelantan, Malaysia \\ [Received 10 November 2012; Accepted 17 December 2012]
}

\begin{abstract}
Anatomical variations in the femoral vein are of great clinical importance especially in cases of deep vein thrombosis (DVT). Knowledge of the variable anatomy of the femoral vein is important to minimise false-negative findings on ultrasound examination in patients with DVT and help to explain the 'silent' DVT. Furthermore, the presence of a duplicated femoral vein itself is associated with higher incidence of DVT. These venous anomalies are usually due to the truncular venous malformation. In the present study, while dissecting the right lower limb, we found a case of variation of the femoral vein. In this case, besides a duplicated femoral vein, we also noticed a $3^{\text {rd }}$ interconnecting channel near the apex of the femoral triangle joining the two veins. This variation has not been reported previously by other authors. Considering its uniqueness and clinical importance, we decided to report this case. (Folia Morphol 2013; 72, 1: 82-85)
\end{abstract}

Key words: femoral vein, deep vein thrombosis, venous malformation, duplication, variation

\begin{abstract}
INTRODUCTION
The venous anatomy of the lower limb is highly variable and these variations have immense clinical significance particularly in cases of deep vein thrombosis (DVT). The femoral vein (FV) throughout its course is normally accompanied by the femoral artery (FA). In the lower part of the adductor canal, the FV lies posterolateral to the FA; while, in the upper part of the canal and in the lower part of the femoral triangle (FT), it is posterior to the FA and immediately below the inguinal ligament, it comes to lie medial to the FA. The FV receives numerous muscular tributaries from the adjacent muscles. One of its largest tributaries, the profunda femoris vein, joins the FV posteriorly, 4-12 cm distal to the inguinal ligament, and the other large tributary, the great saphenous vein, joins it anteriorly. Lateral
\end{abstract}

and medial circumflex FVs are the other tributaries of the FV [14]. The vascular bundle in the FT is utilised for various clinical procedures, both in open and closed interventions, particularly with respect to arterial and venous cannulation, as well as ligation of FVs to prevent the spread of DVT [1]. FV catheterisation is the easiest and safest method for obtaining temporary vascular access in haemodialysis patients.

The complex embryologic development of the vascular system of lower limbs often results in several clinically relevant variants. Most of these variants are due to possible venous malformations, especially minor truncular forms, and these produce several anatomical variations in the number and calibre of the main venous femoral trunks in the thigh [7]. It has been shown that the classic ana-

Address for correspondence: A.A. Khan, Senior Lecturer, Department of Anatomy, Universiti Sains Malaysia, Kubang Kerian-16150, Kelantan, Malaysia, tel: 0060-9-7676080, fax: 00609765 3370, e-mail: aaijazk@gmail.com, aaijaz@kb.usm.my

The study was carried out in the Department of Anatomy, PPSP, USM, Kempus Kesihatan, Kubang Kerian-16150, KotaBharu, Kelantan, Malaysia in the year 2012. 
tomic venous pattern in the lower extremity is found in only $16 \%$ of patients [cited in 5]. Although these variations are part of normal anatomy they may cause difficulties in interpretation of venograms and in operations on veins [2]. During routine dissection of one of the lower limbs, we came across an interesting case of duplication of FV along with another channel connecting these two veins near the apex of the FT. This type of interconnected duplicated FV is a rare occurrence. Various authors used different terms like lateral and medial channels [9], duplicated vessel or duplicated superficial FV [11], or superficial and deep component [10] to describe the duplicated FV. Furthermore, various clinical trials on DVT have suggested that there is a strong relationship between duplicated FV and DVT $[7,11]$. Therefore, due to its immense clinical importance we decided to report this case.

\section{CASE REPORT}

While dissecting the anterior aspect of a right thigh of an intact formaldehyde-preserved male cadaver in the department of Anatomy, School of Medical Sciences, Universiti Sains Malaysia, we noticed that the FV was located in a more posterior plane in relation to the FA. While tracing it further near the apex of the FT and adductor canal we noticed a duplication of the FV.

After exposing the adductor canal we found that the FV was located posterolateral to the FA in the lower part of the canal. In the middle part of the canal, about two inches from the adductor hiatus, we noticed the duplication of the FV, with an intervening FA passing between them (Fig. 1). While tracing further proximally in the adductor canal, we noticed that the duplicated vein wound around the medial aspect of the FA and came to lie on its anterior aspect; while the FV ascended as usual posterior to the FA. In the lower part of the FT, approximately two inches above its apex, the duplicate vein passed around the anterolateral aspect of the FA to unite with the FV, posterior to the FA (Fig. 1). At this junction, as well as these veins, a third vein draining the vastus muscle was also seen joining the FV. The common FV thus formed, coursed a little obliquely, first posterior to the FA, and then near the inguinal ligament it came to lie in its usual medial position. The great saphenous vein and profunda femoris vein (deep FV) joined the FV as usual in the upper part of the FT. We also observed that just above the apex of the FT the duplicated vein and FV were connected by a small interconnecting channel winding on the me-

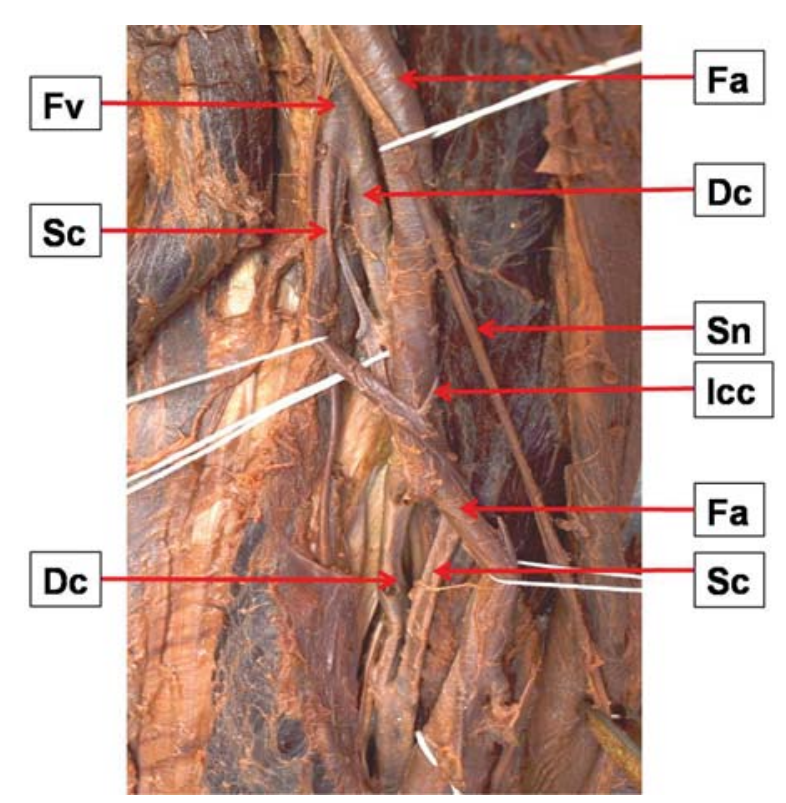

Figure 1. Photograph of the femoral triangle showing duplicated femoral vein (Fv); $\mathrm{Fa}$ - femoral artery; $\mathrm{Sn}$ - saphenous nerve; Dc — deep component; Sc — superficial component; Icc — interconnecting channel.

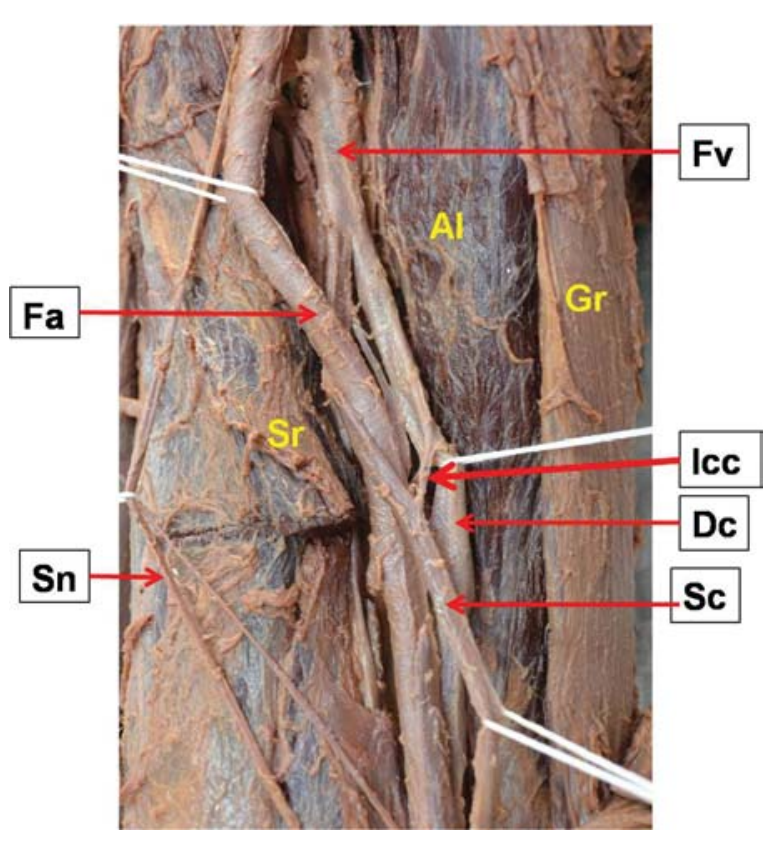

Figure 2. Photograph of the femoral triangle showing interconnecting channel connecting duplicated femoral vein $(\mathrm{Fv}) ; \mathrm{Fa}-$ femoral artery; $\mathrm{Sn}$ - saphenous nerve; Dc — deep component; Sc — superficial component; Icc — inter connecting channel; $\mathrm{Al}$ — adductor longus; $\mathrm{Sr}$ — sartorius; $\mathrm{Gr}$ — gracilis.

dial aspect of the FA (Fig. 2). This type of interconnecting channel has not been reported by other authors who have described similar types of variations. The diameter of the FV near the inguinal ligament was approximately $28 \mathrm{~mm}$. The diameter of the du- 
plicated vein and FV was measured as $9 \mathrm{~mm}$ and $14 \mathrm{~mm}$, respectively. This vascular bundle in the adductor canal and FT was accompanied by the saphenous nerve as usual.

\section{DISCUSSION}

According to embryological classification of venous malformations in the lower limb, 'truncular' venous malformations represent one of the two different types of venous malformations, classified based on the stage at which the developmental arrest or defect occurrs. The truncular venous malformations represent an embryologically defective vein in which developmental arrest has occurred during the vascular trunk formation period in the 'later stage' of embryonic development. This developmental arrest involves the already formed and established venous trunk to varying degrees. On the other hand, extratruncular venous malformations represent defective veins in which developmental arrest occurs in the early stages of development [7]. Considering the above description, our case of duplication of FV seems to be a truncular venous malformation.

The incidence of duplication of FV is quite variable and may reach as high as 46\% [11]. Kerr et al. [6], in their study on venous and arterial anomalies of the lower extremities diagnosed by duplex scanning, noted that duplication of the superficial FV was the most common anomaly of the venous system of lower extremities. Gordon et al. [4] reported the incidence of duplication of the FV in $25 \%$ of their investigated cases. They also noted that the presence of duplication was associated with a significant reduction in the diameter of both limbs of the FV when compared to a normal vessel. We also noticed a reduction in the diameter of two veins $(9$ and $14 \mathrm{~mm}$, respectively) as compared to the trunk $(28 \mathrm{~mm})$. Similarly, Quinlan et al. [11] in their retrospective review of 404 bilateral (808 limbs) lower limb venograms obtained from patients participating in a thromboprophylaxis clinical trial, found the incidence of duplicated superficial FV in 253 (31\%) of limbs. They also observed that the duplicated vein began in the adductor region in only 80 limbs.

Furthermore, Uhl et al. [16], in their dissection study on fresh non-embalmed cadavers, reported that venous malformations (truncular forms) occurring during the late development of the embryo produced several anatomical variations in the number and calibre of main venous femoral trunks at the thigh level. They concluded that the normal anatomy of the FV was found in 308 of 336 limbs (88\%), truncular malformations were found in 28 of 336 limbs (12\%), unitruncular configurations in $3 \%$, and bitruncular configurations in $9 \%$. Although truncular venous malformations of the FV are not rare (12\%), knowledge about them is important for investigation of the venous network, particularly the venous mapping of patients with cardiovascular disease.

A number of medical specialists, especially radiologists, have described a relationship between the presence of multiple FV and the occurrence of DVT. Liu et al. [8] conducted ascending positive contrast venography on 337 lower extremities to determine whether there were any anatomic variations that might predispose to (DVT), and to investigate why so many patients with DVT were asymptomatic. They found multiple $\mathrm{FVs}$ in $31 \%$ of the total limbs. Of the limbs with multiple FVs, $40 \%$ had DVT. This was a statistically higher incidence $(p<0.001)$ than that seen in the $19 \%$ of those limbs with a single FV. They concluded that one of the factors responsible for "silent" DVT is multiple femoral veins. Similarly, Screaton et al. [12] in their retrospective review of 381 venograms found that the number of false-negative ultrasound findings of thrombosis was larger in the presence of a duplicated femoro-popliteal venous system. Liu et al. [8] found that false-negative ultrasound findings occurred in $4(2 \%)$ of 204 patients with single FV and in $10(6 \%)$ of 177 patients with duplicated FVs. They made an important inference that the frequency of missed proximal thrombosis at ultrasound appears to be increased when duplicated FVs are present.

Furthermore, Dona et al. [3] also confirmed a significantly high incidence of duplications of the popliteal vein and the FV and the increased potential for DVT formation secondary to changes in flow velocities. Perhaps the high incidence of DVT in multiple FVs can be explained by increased blood volume in the venous pool and conversely a decreased flow rate, which probably predisposes the limb to DVT $[13,14]$.

More recently, Sharma and Salwan [10] reported a more or less similar type of case of duplication of the FV, but in their case there was no interconnecting channel as noted in our case. Moreover, in the present case the $\mathrm{FV}$ was located more posterior to the FA, and the difference in the diameter of the two components was more as compare to their case.

In the literature review, we noticed that there are differences in opinion regarding the terminology used for FV. Some specialists, especially radiolo- 
gists, orthopaedic, and vascular surgeons use the term "superficial femoral vein" for the initial segment of the FV before joining the profunda femoris vein. In fact, they use the term deep FV for the "profunda femoris vein". From an anatomical perspective, this particular segment of $\mathrm{FV}$ being described as superficial is in fact quite deeply situated both in the FT and in the adductor canal and therefore does not fit the criteria of superficial vein. Furthermore, this incorrect term does not appear in any definitive anatomic textbooks including Gray's anatomy, which is considered the most authenticate textbook of anatomy [15]. Therefore, it should "not" be recognised as a legitimate term. According to Hammond [5], confusion arising from the use of such an inappropriate term may be a reason for many cases of clinical mismanagement and death as patients (with DVT of FV) are denied efficacious thrombolytic therapy. Therefore, the misleading term 'superficial femoral' vein should never be used in place of the FV, as it is definitely a deep vein and is not a part of the superficial venous system.

\section{CONCLUSIONS}

To summarise, we can put forth the contention that though truncular venous malformations of the $\mathrm{FV}$ are not rare phenomenon but the knowledge of their occurrence is very important for investigation of the lower limb venous network, especially for venous mapping in patients with cardiovascular diseases, and to avoid potential errors in the diagnosis of DVT of the FV and in the case of an occluded duplicated trunk in bitruncular configuration. Radiologists should also keep in mind the possible presence of a duplicated FV while performing investigations in cases of suspected proximal DVT otherwise they can miss the diagnosis. Similarly, vascular surgeons can also take advantage of duplicated FV, as they can use one of these trunks for deep vein transposition reconstruction surgery on the opposite side of the limb.

\section{ACKNOWLEDGEMENTS}

I am very grateful to the people who are instrumental in the preparation of this manuscript. I am thankful to the Department of Anatomy technologist Norhana binti Arshad, Mohd Eidi Azhary, En.Hafiz, and secretary Nik Fadzlina binti Nik Mohd
Hanafi for their support and help in cadaveric dissection and photography.

\section{REFERENCES}

1. Bandyopadhyay M, Biswas S, Roy R (2010) Vessels in femoral triangle in a rare relationship. Singapore Med J, 51: 3-5.

2. Cockett FB (1954) Abnormalities of the deep veins of the leg. Post Med J, 30: 512-522.

3. Dona E, Fletcher JP, Hughes TM, Saker K, Batiste P, Ramanathan I (2000) Duplicated popliteal and superficial femoral veins: incidence and potential significance. Aust N Z J Surg, 70: 438-440.

4. Gordon AC, Wright I, Pugh ND (1996) Duplication of the superficial femoral vein: recognition with duplex ultrasonography. Clin Radiol, 51: 622-624.

5. Hammond I (2003) The superficial femoral vein. Radiology, 229: 604-606.

6. Kerr TM, Smith JM, McKenna P, Lutter KS, Sampson MG, Helmchen RH, Roedersheimer LR (1992) Venous and arterial anomalies of the lower extremities diagnosed by duplex scanning. Surg Gynecol Obstet, 175 : 309-314.

7. Lee BB, Laredo J, Neville R (2010) Embryological background of truncular venous malformation in the extracranial venous pathways as the cause of chronic cerebrospinal venous insufficiency. Int Angiol, 29: 95-108.

8. Liu GC, Ferris EJ, Reifsteck JR, Baker ME (1986) Effect of anatomic variations on deep vein thrombosis of the lower extremity. Am J Roentgenol, 146: 845-848.

9. Park EA, Chung JW, Lee W, Yin YH, Ha J, Kim SJ and Park JH (2011) Three-dimensional evaluation of the anatomic variations of the femoral vein and popliteal vein in relation to the accompanying artery by using CT venography. Korean J Radiol, 12: 327-340.

10. Sharma P, Salwan S (2011) Duplication of femoral vein and its significant clinical implications. IJAV, 4: 188-191.

11. Quinlan DJ, Alikhan R, Gishen P, Sidhu PS (2003) Variations in lower limb venous anatomy: implications for US diagnosis of deep vein thrombosis. Radiology, 228: 443-448.

12. Screaton NJ, Gillard JH, Berman LH, Kemp PM (1998) Duplicated superficial femoral veins: a source of error in the sonographic investigation of deep vein thrombosis. Radiology, 206: 397-401.

13. Sigel B, Edelstein AL, Felix WR Jr, Memhardt CR (1973) Compression of the deep venous system of the lower leg during inactive recumbency. Arch Surg, 106: 38-43.

14. Sevitt S (1960) Etiology and pathogenesis of deep vein thrombosis. Lancet, 275: 384-385.

15. Standring $S$ ed. Gray's anatomy. $39^{\text {th }}$ Ed. Elsevier. London, 2005: 543-547.

16. Uhl JF, Gillot C, Chahim M (2010) Anatomical variations of the femoral vein. J Vasc Surg, 52: 714-719. 